\title{
Beam Energy Absolute Measurement Using K-Edge Absorption Spectrometers
}

Ivan P. Karabekovt and George R. Neilł

†Yerevan Physics Institute

2 Alikhanian Brothers st., Yerevan, 375036, Republic of Armenia

$\mp$ Continuous Electron Beam Accelerator Facility

12000 Jefferson Avenue

Newport News, VA 23606

\section{Continuous}

\section{ELECTRON}

BEAM

AcCelerator

FACILITY

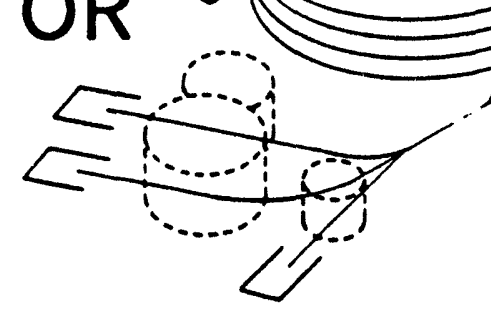

SURA SOUTHEASTERN UNIVERSTIES RESEARCH ASSOCIATION

CEBAF

Newport News, Virginis 
Copies available from:

\author{
Library \\ CEBAF \\ 12000 Jefferson Avenue \\ Newport News \\ Virginia 23606
}

The Southeastern Universities Research Association (SURA) operates the Continuous Electron Beam Accelerator Facility for the United States Department of Energy under contract DE-AC05-84ER40150.

\title{
DISCLAIMER
}

This report was prepared as an account of work oponoored by the United Statea government. Neither the United States nor the United States Department of Energy, nor any of their employees, makes any warranty, express or implied, or assumes any legal liebility or responsibility for the accuracy, completeness, or usefulness of any informati or, apparatus, product, or procese dieclosed, or represents that its use would not infringe privately owned rights. Reference herein to any opecific commercial product, proceas, or service by trade name, mark, manufacturer, or otherwise, does not necessarily conotitute or imply its endorsement, recommendation, or favoring by the United States government or any asency thereof. The viewe and opinions of authore expressed herein do not necesearily state or reflect those of the United States government or any agency thereof. 


\author{
Beam Einergy Absolute Measurement Using \\ K-Edge Absorption Spectrometers* \\ Ivan P. Karabekov $\nmid$ and George R. Neil $\nmid$ \\ †Yerevan Physics Institute \\ 2 Alikhanian Brothers st., Yerevan, 375036, Republic of Armenia \\ $\ddagger$ Continuous Electron Beam Accelerator Facility \\ 12000 Jefferson Avenue, Newport News, Virginia 23606
}

\begin{abstract}
A method is presented of absolute energy measurement with an accuracy of $\Delta E \sim$ $10^{-4} E_{0}$ by direct measurement of the bend angle in a high-precision magnetic dipole using two opposite-direction short (about $2 \mathrm{~mm}$ long) high-field-intensity magnets $\left(\bar{B}_{\text {dipole }} \ll\right.$ $B_{\text {short mag }}$ ) installed at each end and two K-edge absorption spectrometers. Using these spectrometers and the hard $\mathrm{x}$-ray synchrotron radiation created by the short magnets, a bend angle of 4.5 arc deg for the CEBAF energy bandwidth can be measured with an accuracy of a few units of $10^{-6} \mathrm{rad}$, and the main sources of systematic errors are the absolute measurement of the field integral and the determination of the centroid of the synchrotron beam at a wavelength equal to the K-edge absorption of the chosen substance.
\end{abstract}

\title{
Introduction
}

The use of magnetic spectrometers to determine absolute beam energy is well known. The best performance of the method was the SLC energy measurement facility [1]. This facility measured the bending angle using two synchrotron radiation (SR) swaths created in two short kicker magnets having magnetic field perpendicular to the field direction in the main bending magnet and installed on its sides. The field integral $\int B d l$ in the main dipole was measured with an accuracy better than $10^{-4}$ and monitored by a special rotating coil or NMR probes [2].

Two phosphorescent screens equipped with a system of fiducial wires and CCD video cameras measured the distance between SR beams with an accuracy of $\leq 10^{-4}$.

The distance between the center of the main bending and the phosphorescent screen equaling $15 \mathrm{~m}$ was measured with an absolute accuracy of $1.5 \mathrm{~mm}$.

The main source of errors limiting the accuracy of energy determination at the level of $5 \times 10^{-4}$ is nonorthogonality of the vectors of the magnetic field strength in the kickers and in the main bending dipole [3].

In this paper a new method of bend angle measurement using the K-edge absorption technique is discussed. The application of the method to high-precision absolute energy measurement at CEBAF is presented.

* Work oupported by U.S. Department of Energy under contract DE-AC05-84ER40150. 


\section{The method}

The concept of the method is the following. A beam of electrons passes through a high-precision mapped and continuously monitored dipole magnet having a field average intensity $\bar{B}_{b}=\frac{1}{\Delta l} \int^{\Delta l} B d l$ and two short dipoles having opposite direction of field intensity $B, \gg \bar{B}_{b}$ installed on its sides. The synchrotron radiation generated in the main dipole and short magnets have different spectral distributions defined by corresponding critical wavelengths $\lambda_{c_{b}}$ and $\lambda_{c_{0}}$ which are chosen to satisfy the condition $\lambda_{c_{b}} \gg \lambda_{c_{0}}$. So these short magnets are working as magnetic targets generating wavelength $\lambda_{0}$, which is practically not represented in the spectrum radiated from electrons in the main dipole.

The principle of the method is as follows. Two K-edge absorption spectrometers (see Figure 1) having input collimators of width $h$ and installed on the SR beam produced by the first short magnet at distance $L$ can be tuned up at a wavelength $\lambda_{0}$ between the upper and lower borders of K-edge absorption of a chosen element. Scanning synchrotron light by the input collimator one can find the higher intensity at chosen wavelength which is the centroid of the SR beam generated in the first short magnet. This procedure will lower the quantum fluctuation of output (after absorption) intensity, which is one of the main sources of systematic errors. Then one of the spectrometers will be installed on the second beam axis by moving in a direction perpendicular to the axis of the first SR beam. The same wavelength $\lambda_{0}$ with the same output intensity can be registered in the SR beam generated in the second short magnet by scanning this beam and rotating the second spectrometer around the axis of its first crystal. When the same point of the absorption curve is reached-i.e., when the output intensities $N_{\text {yout }}$ of both spectrometers are equal and the gradients $\Delta N_{\text {yout }} / \Delta \theta$ of both spectrometers are equal-the bend angle $\theta_{b}$ in the main dipole will be equal to the angle of rotation of the second spectrometer.
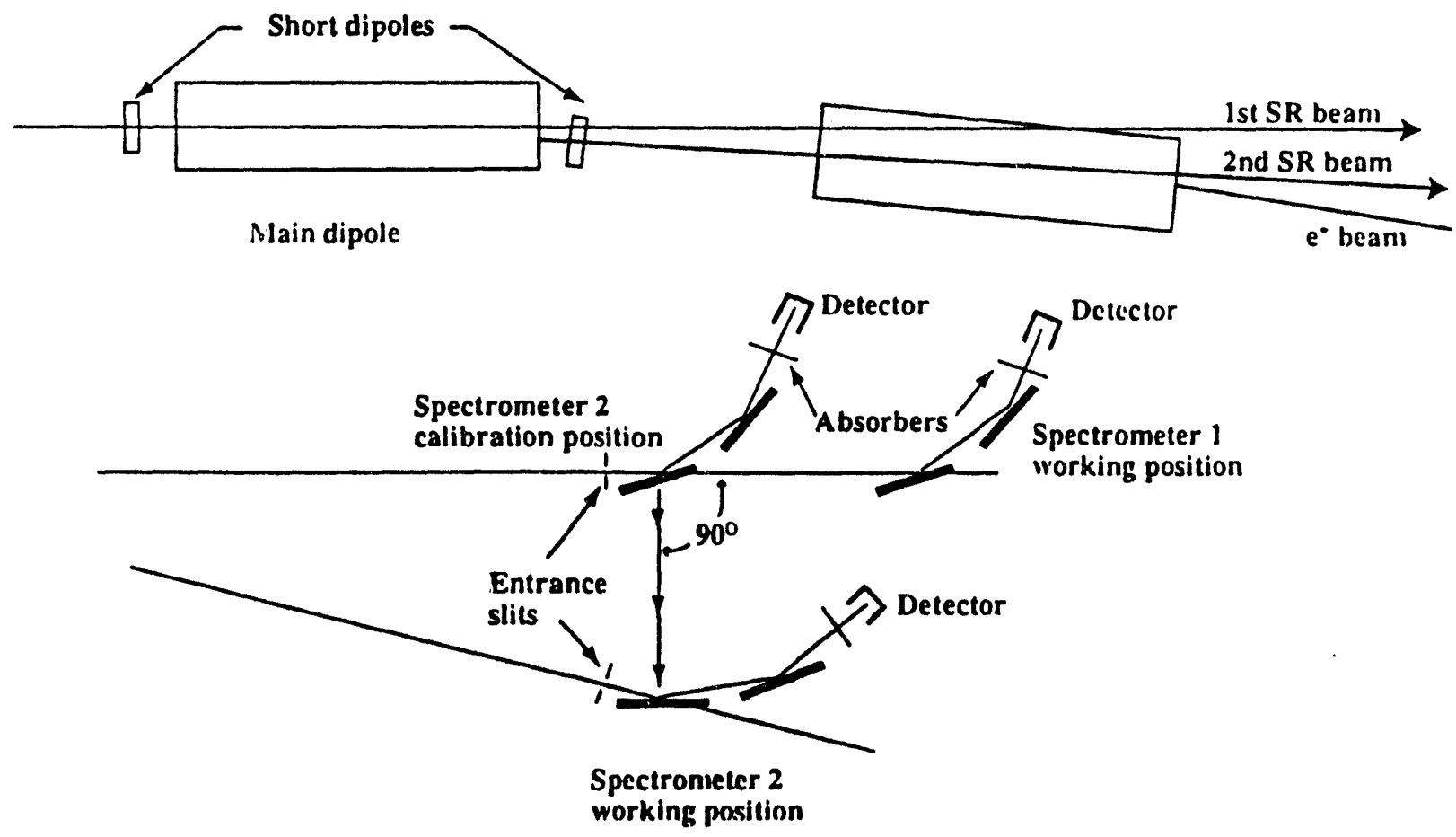

Figure 1. The scheme of absolute energy measurement with the K-edge spectrometers. 
The accuracy of the measurement depends on the angle of SR beam collimation, $h / L$, and the absolute value of the intensity drop gradient $\Delta N_{\text {yout }} / \Delta \theta$ between the upper and lower points of the absorption curve.

To exclude from $\theta_{b}$ the bend angle created by the short dipoles $\delta \theta$, these magnets have opposite directions of the vectors of magnetic field strength.

For the spectrometers, ordinary EXAFS (extended x-ray absorption fine structure) spectrometers can be used (see, for example, $[4,5]$ ). Double crystal monochromators which are in use for these spectrometers have an angular resolution better than 1 arc sec. The monochromator described in [4] consists of two ron-dispersive $\mathrm{Si}(422)$ channel-cut crystals, whose rocking curve width is $\varepsilon \sim 2.6$ arc sec, installed in a dispersive geometry (see Figure 2) having an energy resolution $\Delta E_{\gamma} / E_{\gamma 0}$ better than $10^{-5}$ and permitting measurement of the angle of about $5.6 \operatorname{arc} \operatorname{deg}$ from $\mathrm{CuK} \alpha_{1,2}$ to $\mathrm{CuK}_{\beta}$ with an accuracy $\delta \phi$ better than $0.6 \mathrm{arc} \mathrm{sec}$. This accuracy was stable in several sweeps over a period of several hours.

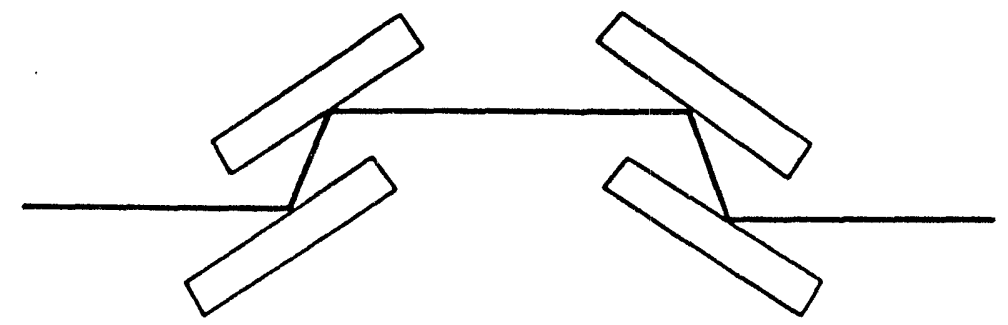

Figure 2 High-resolution crystal monochromator for EXAFS spectrometer.

Using this type of spectrometer the bend angle $\phi$ of the electron beam in the main dipole (about 4.5 arc deg) will be measured with the accuracy $\delta \phi / \phi$ better than $4 \times 10^{-5}$.

\section{The calculations}

The angular resolution of the K-edge spectrometer depends on the absolute value of a gradient of the intensity drop within the upper and lower $\left(\lambda_{u}\right.$ and $\left.\lambda_{l}\right)$ borders of K-edge absorption:

$$
G=\frac{N_{\gamma} l-N_{\gamma} u}{\Delta \phi},
$$

and the intensity fluctuation at the lower border of absorption $N_{f l}=\sqrt{N_{\gamma} l}$, at a given electron beam intensity, $I_{e}$, and can be determined as follows:

$$
\delta \phi \times G=\sqrt{N_{\gamma} l} .
$$

The gradient of intensity drop depends on a ratio between the intensities created at $\lambda_{0}$ in the short and main dipoles too, because of the large divergence angle of radiation in the main dipole. This ratio can be limited to a few units of $10^{-2}$.

The magnetic field intensity in the short dipole $B$, can be taken as $2 \mathrm{~T}$ and with a bend angle of $2 \times 10^{-5} \mathrm{rad}$.

A typical absorption curve of $\mathrm{Cu}$ at its $\mathrm{K}$ edge with an energy resolution about $2 \mathrm{eV}$ [5] having the ratio ${ }_{\gamma} u / N_{\gamma} l$ higher than 30 has a $\Delta \phi$ equal to $4.25 \times 10^{-4}$ rad (see Figure $3)$. For the following calculations these parameters were used. 


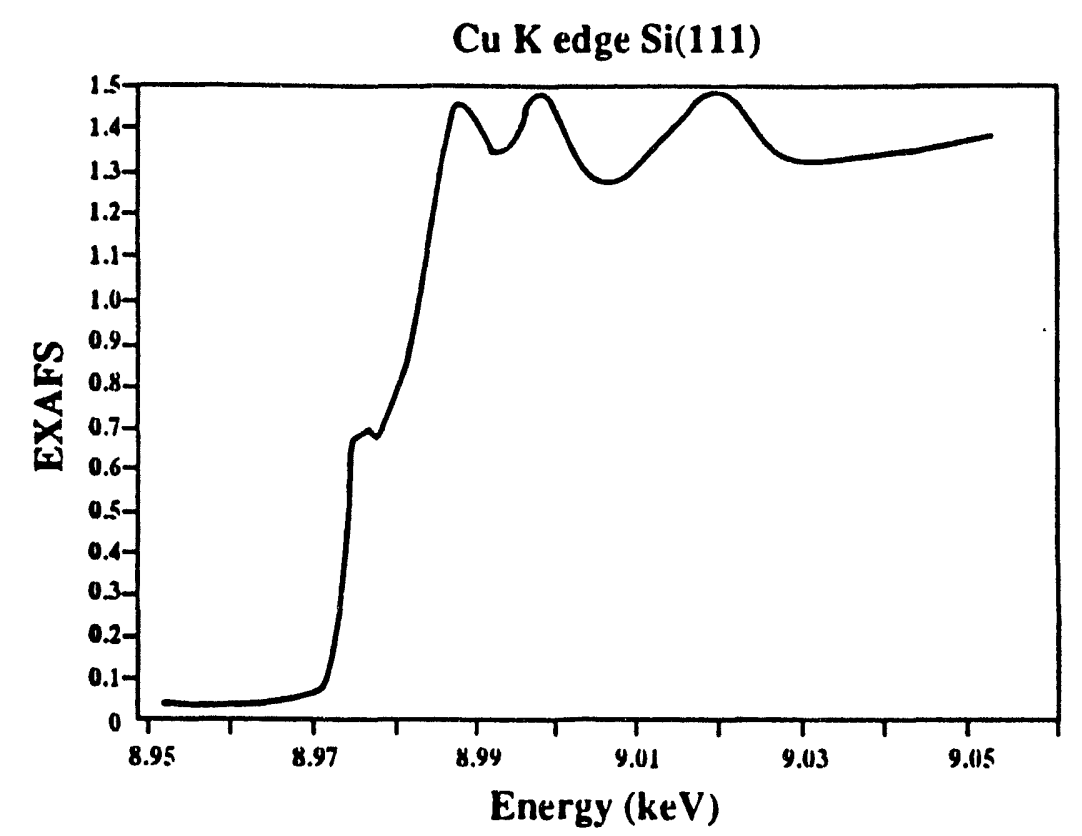

Figure 3. A typical absorption curve of $\mathrm{Cu}$.

The intensity of the photon flux generated in the short magnet at the chosen working wavelength $\lambda_{0}$ was calculated using following formula [6]:

$$
N_{\gamma}=A \gamma^{2}\left(\lambda_{c} / \lambda_{0}\right)^{2}\left(\Delta \lambda / \lambda_{0}\right) K_{2 / 3}^{2}\left(\lambda_{c} / 2 \lambda_{0}\right),
$$

where $K_{2 / 3}\left(\lambda_{c} / 2 \lambda_{0}\right)$ is the modified Bessel function, and $A$ is a proportionality coefficient to obtain the result in units of photons per second:

$$
A=3.461 \times 10^{6} I[\mathrm{~mA}] \phi[\mathrm{mrad}] \psi[\mathrm{mrad}],
$$

where $\phi$ and $\psi$ are horizontal and vertical angles of SR beam collimation and

$$
\lambda_{c}=\frac{7.12 \times 10^{3}}{\gamma^{2} B} .
$$

Here $\gamma$ is Lorentz factor of the electron.

The ratio of the intensities generated at the wavelength $\lambda_{0}$ in the main bending and short magnets can be calculated as follows:

$$
R=\left(\frac{\lambda_{c_{b}}}{\lambda_{c_{0}}}\right)^{2} \times \frac{K_{2 / 3}^{2}\left(\lambda_{c_{0}} / 2 \lambda_{0}\right)}{K_{2 / 3}^{2}\left(\lambda_{c_{0}} / 2 \lambda_{0}\right)}
$$
used.

For optimization of this parameter at all CEBAF energies different substances can be 
The calculations which affirm the high resolution of the method for CEBAF were performed using the following parameters:

* beam current: $10 \mu \mathrm{A}$,

* bend angle in main dipole: $8.17 \times 10^{-2} \mathrm{rad}$,

* magnetic field intensity in short magnets: $2 \mathrm{~T}$,

* SR beam horizontal divergence from short magnet limited by collimator: $2 \times 10^{-5}$ $\mathrm{rad}$

* SR beam vertical divergence limited by collimator: $1 \times 10^{-3} \mathrm{rad}$,

* the working wavelengths, $\lambda_{0}$, are K-edges of the following substances: $\mathrm{Cu}(1.38059$ $\AA)$, for an accelerator beam energy from 1 and $2 \mathrm{GeV}, \mathrm{Ge}(1.11658 \AA)$ for $3 \mathrm{GeV}$, and Mo $(0.61978 \AA)$ for $4 \mathrm{GeV}$,

* spectral bandwidth, $\Delta \lambda / \lambda: 2 \times 10^{-4}$, and

* the angular bandwidth, $\Delta \phi$, of the intensity drop at the K-edges: $4.5 \times 10^{-5} \mathrm{rad}$ for copper, $2 \times 10^{-5} \mathrm{rad}$ for germanium, and $1 \times 10^{-5} \mathrm{rad}$ for molybdenum.

The results of the calculations are presented in Table 1.

Table 1. The Results of the Calculations

\begin{tabular}{|c|c|c|c|c|c|c|}
\hline $\begin{array}{l}E_{e} \\
(\mathrm{GeV})\end{array}$ & $\begin{array}{c}\bar{B}_{b} \\
(\mathrm{~T})\end{array}$ & $\begin{array}{c}\lambda_{c_{b}} \\
\AA\end{array}$ & $\begin{array}{c}\lambda_{c_{0}} \\
\AA\end{array}$ & $\begin{array}{c}N_{\gamma_{\iota}} \\
\text { photons/sec }\end{array}$ & $N_{\gamma_{b}} / N_{\gamma_{\iota}}$ & $\begin{array}{c}\Delta N_{\gamma} / \Delta \theta \\
\text { phot. } \mathrm{sec}^{-1}, \mathrm{rad}^{-1}\end{array}$ \\
\hline 1.0 & 0.0907 & 205.0 & 9.3 & $1.385 \times 10^{4}$ & $<10^{-5}$ & $3.06 \times 10^{8}$ \\
2.0 & 0.1814 & 25.62 & 2.3 & $2.532 \times 10^{6}$ & $<10^{-5}$ & $5.62 \times 10^{10}$ \\
3.0 & 2.721 & 7.59 & 1.03 & $1.86 \times 10^{6}$ & $1.37 \times 10^{-2}$ & $9.3 \times 10^{10}$ \\
4.0 & 0.3625 & 3.2 & 0.58 & $1.253 \times 10^{7}$ & $6.84 \times 10^{-2}$ & $1.253 \times 10^{12}$ \\
\hline
\end{tabular}

To avoid the influence of harmonics $\lambda_{0} / n$ on the gradient of intensity drop, total external reflection (TER) will be used. The spectrum transformation by silicon TER mirror taken from [7] is presented in Figure 4.
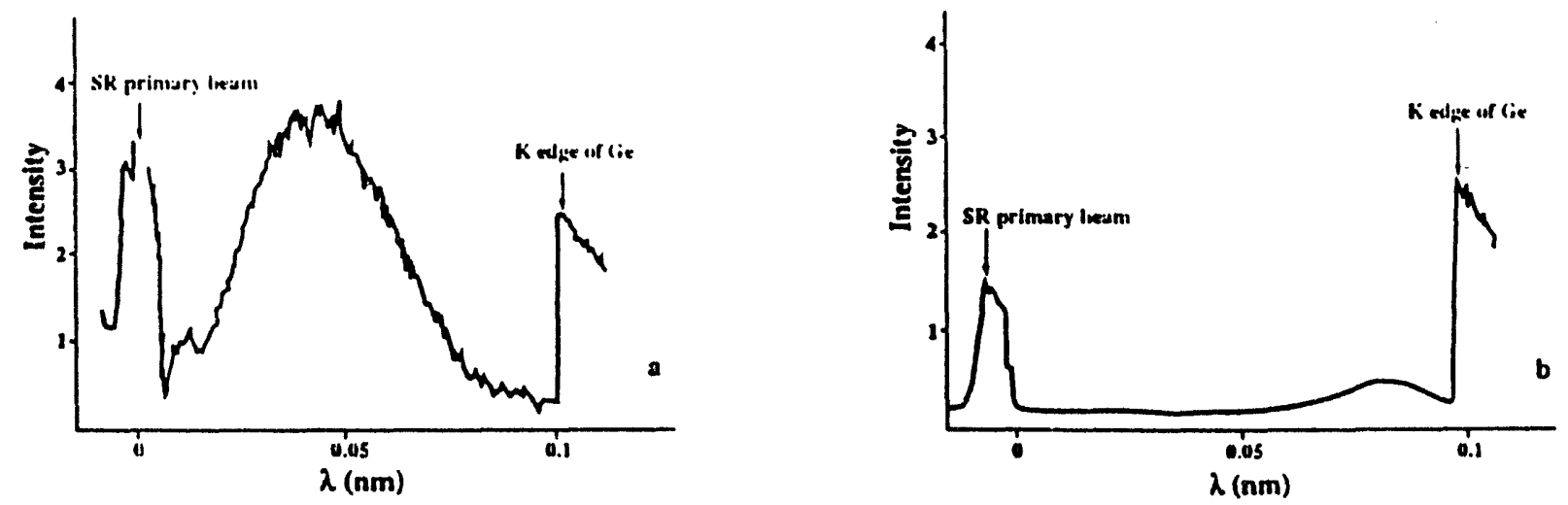

Figure 4. The synchrotron radiation spectrum transformation by silicon TER mirror.

According to Table 1 and formula (2) the angular resolution $\delta \phi$ is better than 1 arc sec, and the limitation of absolute energy determination has mainly equal contributions from the accuracy of the magnetic field mapping in the main dipole, i.e., about $10^{-4}$. 
The intensity at the lower border of K-edge absorption according to [5] is taken equal to $N_{\gamma_{o}} / 30$.

For CEBAF Halls $\mathrm{A}$ and $\mathrm{C}$ one bending magnet in each beam transport line is used. Two short ( $2 \mathrm{~mm}$ long) magnets installed at both ends of the dipole could generate SR beams extracted through a beryllium window of a modified electron beam pipe into two EXAFS spectrometers.

\section{Acknowledgment}

We would like to thank Dr. J. Bisognano for very valuable discussions.

\section{References}

1. J. Kent, M. King, C. Von Zanthier, S. Watson, "Precision measurement of the SLS beam energy", SLAC-PUB-4922, 1989.

2. M. Levi, "Precision measurement of the SLC spectrometer magnets", SLAC-PUB$4654,1989$.

3. M. Levi and F. Rouse, "Precision synchrotron radiation detectors", SLAC-PUB$4921,1989$.

4. Helio Tolento and Ricardo Rodrigues, Rev. Sci. Instrum. 63(1) 946-949, January 1992.

5. Yaning Xie, Hai Yang, Qiao Shan, Shaojian Xia, and D. Xian, Rev. Sci. Instrum. 63(1), 956-957, January 1992.

6. G. K. Green, "Spectra and optics of synchrotron radiation", BNL-50522, April 1976.

7. I. P. Karabekov, D. L. Egikian, R. A. Mikaelian, and V. G. Bagdasarian, Nucl. Instrum. Methods A261(1987) 318-321. 
$1 !$
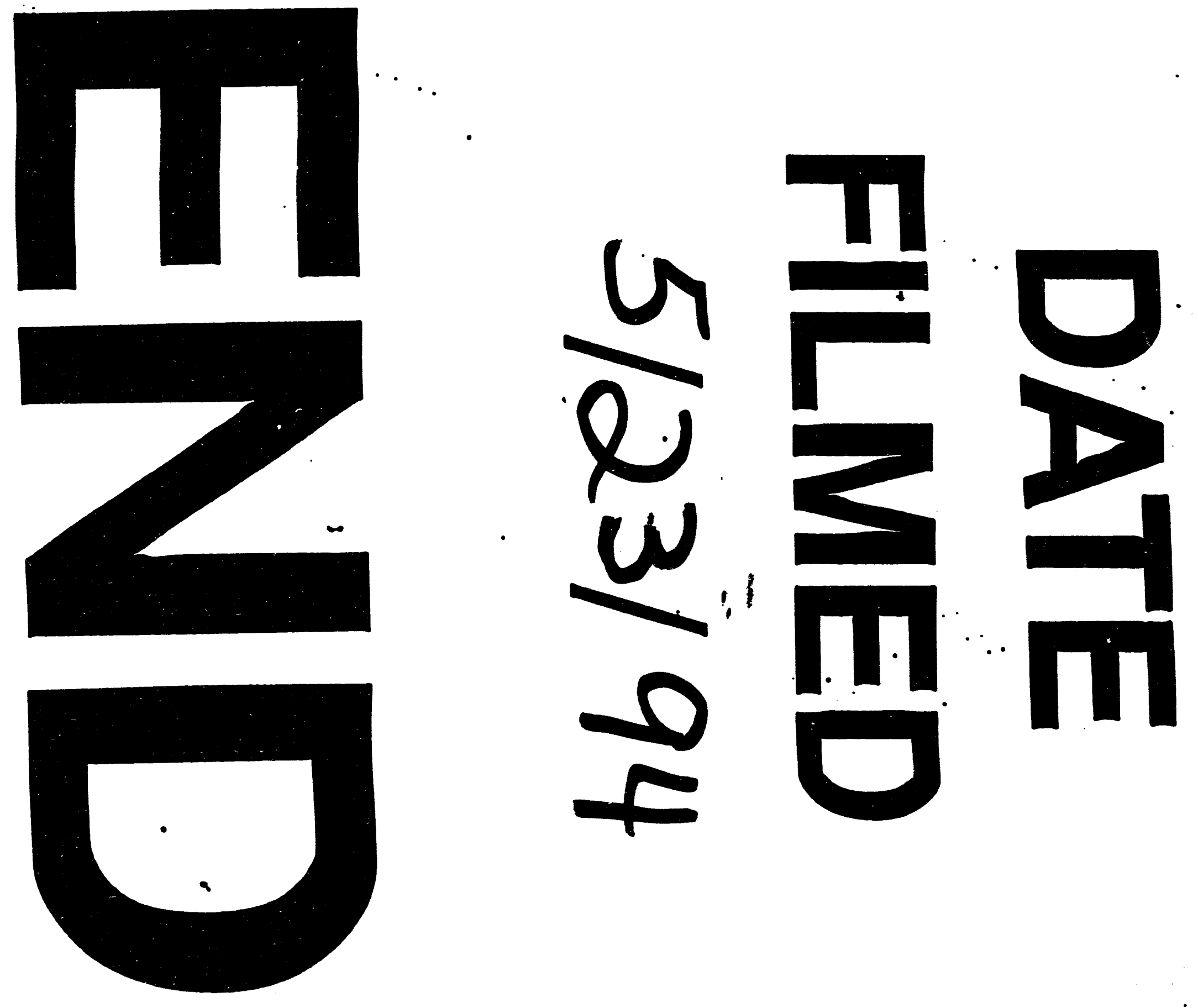
